\title{
1 Red deer Cervus elaphus blink more in larger groups
}

3 Zeke W. Rowe*, Joseph H. Robins* and Sean A. Rands $†$

5 School of Biological Sciences, University of Bristol, Life Sciences Building, Tyndall Avenue,

6 Bristol BS8 1TQ, United Kingdom

8 ORCID: ZWR: 0000-0002-9617-5539

9 JHR: 0000-0001-8980-8150

12 * JR and ZR are considered as joint first authors

14 † author for correspondence: sean.rands@bristol.ac.uk

\section{ABSTRACT}

Most animals need to spend time being vigilant for predators, at the expense of other activities such as foraging. Group-living animals can benefit from the shared vigilance effort of other group members, with individuals reducing personal vigilance effort as group size increases. Behaviours like active scanning or head lifting are usually used to quantify vigilance, but may not be accurate measures as the individual could be conducting them for

24 other purposes. We suggest that measuring an animal's blinking rate gives a meaningful measure of vigilance: increased blinking implies reduced vigilance, as the animal cannot detect predators when its eyes are closed. We demonstrate that as group size increases in red deer, individuals increase their blink rate, confirming the prediction that vigilance should decrease. Blinking is a simple non-invasive measure, and offers a useful metric for assessing the welfare of animals experiencing an increase in perceived predation risk or other stressors. 


\section{INTRODUCTION}

34 Most animal species spend some part of their lives aggregated together in groups, and

35 many benefits have been proposed and tested for this behaviour [1,2]. For prey species,

36 grouping behaviour can offer protection from predators through both the dilution of individual

37 risk if an attack occurs [3-5] and an increase in the chance of successfully detecting an

38 approaching predator due to the combined vigilance effort of the group [5-7], along with

39 other anti-predator advantages of grouping behaviour such as synchronising activity to dilute

40 risk [8-11]. If an animal is being actively vigilant, it may be unable to conduct (or less

41 efficient at) other important behaviours (like foraging or resting) at the same time (e.g. [12]).

42 Group membership means that vigilance can be pooled among the group members, which

43 could mean that each individual can spend less time being vigilant and more time

44 conducting other fitness-enhancing behaviours. A rich body of theory and research has

45 explored how group size and individual vigilance effort are related [13-16], with much of it

46 focussing on the prediction that individual vigilance effort will decrease as the group

47 becomes larger. This prediction requires each individual to show a trade-off between

48 vigilance and other behaviours, where being actively vigilant either cannot occur at the same

49 time as other behaviours, or leads to a reduction in the efficiency of other behaviours that

50 are conducted at the same time as being vigilant.

51

52 Vigilance is usually assumed to be occurring when an animal is actively scanning its 53 surrounding environment with its head upwards, although there is no obvious consensus in

54 how vigilance is defined in any particular species (see [17] for discussion of this problem in

55 studies on primates). Although scanning behaviour is likely to stop an animal from actively

56 collecting food, this head-up activity may not completely interfere with simultaneous

57 conducted behaviours, such as chewing or social interaction. If a behaviour that is recorded

58 as vigilance allows an individual to do other things at the same time, then we may be falsely

59 assuming that this behaviour incurs the time and attention costs that are associated with

60 vigilance [18]. Without careful experimentation, it is difficult to assess how much of an

61 individual's attention is devoted to vigilance when we observe scanning or other forms of

62 vigilance-like behaviour, which may add to the huge variation (e.g. [13]) in whether a study

63 demonstrates that individual vigilance is related to group size or not.

65 Although it is difficult to define exactly when an individual is being vigilant, we may instead

66 be able to define when it is not able to be vigilant. Blinking (the temporary closure of both

67 eyes, involving movements of the eyelids [19]) is a good example of an activity where an

68 individual is momentarily unable to visually scan the environment. It is an essential 
maintenance behaviour to keep the eyes moist and clean [20], and is conducted tens of times every minute in some species of diurnal mammals [21-23] and birds [24]. Although a blink takes only a fraction of a second, the sum of this loss of visual information over multiple blinks could be substantial for the individual. In humans, spontaneous blinking is accompanied by attentional suppression, where the individual experiences a blackout in visual attention for the duration of the blink, meaning that there is no awareness of the temporary blindness and lack of visual information whilst the blinking is occurring $[25,26]$. Blinking suppresses activity in both the visual cortex and other areas of the brain that are associated with awareness of environmental change [27]. If we assume that other animals show similar attentional suppression, then they are essentially blind and unaware of changes in their visual environment during each blink, which in turn means that they cannot be vigilant for predators.

An individual's blink rate therefore presents a trade-off between the physiological benefits of blinking and the loss of visual information during the blink [20]. If an animal needs to dedicate more time to vigilance in a risky environment, then it has to reduce or suppress blinking to accommodate this increased vigilance. This is anecdotally demonstrated in American crows Corvus brachyrhynchos, which reduce their blink rates when looking at potentially dangerous stimuli [28], and in horses Equus caballus, which decrease their spontaneous blink rate in response to stress-inducing stimuli [29]. This link between blink rate and vigilance implies that blink rate will also be related to group size. As group size increases, theory predicts that individual vigilance can be reduced [5], and so any requirement to suppress blinking will be relaxed. Blink rate may therefore increase with an increase in group size. Evidence supporting this is anecdotal: a comparison of chickens Gallus gallus feeding solitarily or in pairs showed a non-significant increase in blink rate in the group-feeding birds [30], while a comparison of the blink rates of olive baboons Papio anubis [31] showed individuals in a small group blinked less than those in a large group (although the two groups were studied in different years). Here, we test this hypothesis by observing the blink rates of group-living red deer Cervus elaphus. Red deer are a prey species that spend most of their lives in dispersed groups, and females are known to reduce vigilance behaviour as group size increases [32,33]. Because they increase vigilance in smaller groups, we therefore predict that they should also reduce their blink rate to avoid losing visual information about their environment. Given that vigilance has been shown to be related to the sex and age of an individual [32,33], we included these individual characteristics in our analysis. 


\section{METHODS}

\section{Study area, time and subjects}

109 This observational study was conducted on the herd of red deer within the 40.5 hectare deer 110 park in Ashton Court Estate, Bristol, England $\left(51.4440^{\circ} \mathrm{N}, 2.6378^{\circ} \mathrm{W}\right)$, which is composed

111 mainly of open grassland, with scattered forestry. The herd, managed by Bristol City

112 Council, consists of c. 110 individuals of varying age and sex, who appear to mix freely. The

113 enclosure is open to the public outside of the rutting season, so the deer are habituated to

114 both dogs and humans. Our observations were conducted over five days during the rutting

115 season; observations were restricted between 1200-1630 h so they were outside of the

116 dawn and dusk peaks of regular rutting activity [34].

118 Behavioural sampling and observations

A random individual was selected as described in previous research on this herd [35]. The selected individual was observed and dichotomously aged (mature or young) and sexed (male or female). The individual was sexed by the presence of antlers, as after one year of age only males have antlers [36]. Age was identified by an individual's size and morphology (larger individuals were older). If the individual was observed suckling, it was discarded and randomisation was repeated, as young individuals are hard to sex and exhibit behaviours uncommon to the rest of the herd [34]. A count of the total number of young/mature males and females, along with suckling young, was made on three different days. The rounded averages of these five demographic classes were calculated, and used in the pseudoreplication analyses presented (see the Supplementary Information).

Prior to the study, the observers (ZWR and JHR, who both conducted the measurements described) were trained in identifying the recorded behaviours, and pilot trials ensured repeatability of measurements. A blink was defined as a rapid full closure of the eye. Group size was arbitrarily defined as the number of individuals aggregated no more than five body lengths away from at least one member of the group containing the focal deer, meaning a measured group was composed of individuals associated by a chain rule of association (see [37] for discussion of defining groups by associated neighbours within arbitrary distances). Before starting any set of observations, the observers waited 10 minutes at the site to habituate the deer. Observations were conducted approximately 10-100 m away from focal individuals using a $30 \times$ zoom spotting scope. An observation for a single selected individual was recorded for a maximum of 10 minutes. At the start of each minute the group size was

142 counted by one observer, with blink rate (blinks per minute) being continuously recorded for 
143 each minute by the other observer. If the deer's eye(s) were obstructed or there was a

144 human/animal disturbance the observations were stopped with the current minute's

145 measures being disregarded. 75 individuals were observed using this method.

\section{Statistical analysis}

Data were recorded as blinks per minute with group size recorded for that minute. Individuals were recorded a mean of 4.9 ( $\pm 2.0 \mathrm{SD})$ minutes before the data collection had to be discontinued due to the observer's view of the eyes being obstructed. For each individual, we calculated the mean number of blinks in a minute, and the mean group size per minute. To compensate for any unevenness caused by some mean values being based on more observations than others, we conducted the same analyses using just the first minute of data for all individuals (see the Supplementary Material): these data gave qualitatively similar results to the analysis involving mean group sizes, and are not discussed further. of mean blink rate was described by the natural logarithm of group size, and the maturity and sex of the focal individual, including the observation date as a random effect. Logarithms were used to satisfy model conditions. A full model including interactions was initially considered, but no interaction terms were significant and so the basic additive model with the three explanatory variables was used. The Supplementary Material describes resampling analysis conducted to explore any effects of pseudoreplication arising from potentially observing the same individuals multiple times.

\section{RESULTS}

171 Blink rate increased with group size $\left(t_{67}=11.38, p<0.001\right.$; figure 1$)$, and adults blinked

172 more than young deer $\left(t_{67}=2.11, p=0.038\right.$; figure 1$)$. There was no relationship between

173 sex and blinking ( $\left.t_{67}=0.35, p=0.727\right)$. Model estimates and effect sizes are given in the

174 Supplementary Material, along with analyses demonstrating that pseudoreplication is

175 unlikely to have influenced the group size effect. 


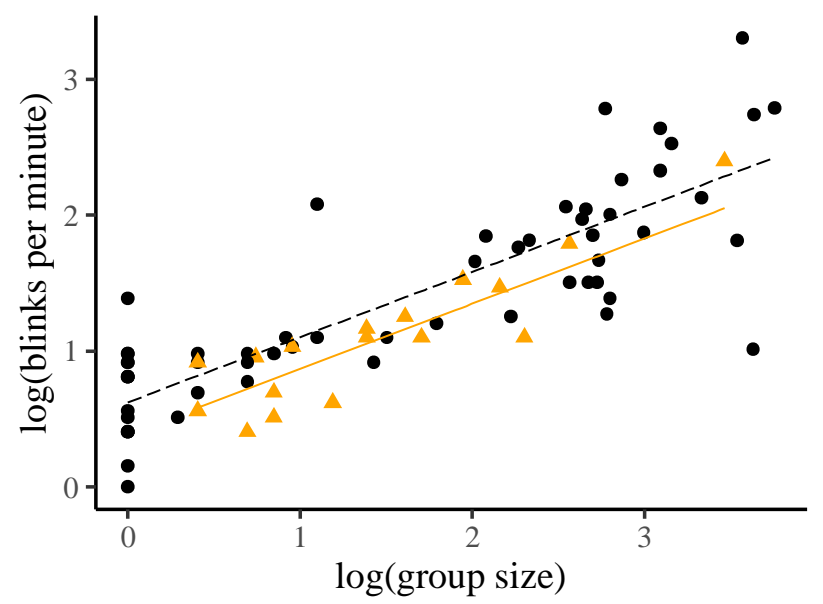

179 Figure 1. Scatterplot showing that blink rate increases with group size and maturity (where 180 orange triangles and the fitted solid line represent young individuals, and black circles and 181 the fitted dashed line represent adults).

\section{DISCUSSION}

Our results demonstrate that blinking increases as group size increases. Given that blinking interferes with vigilance behaviour, and that individual vigilance is predicted to decrease as groups get larger, this supports our argument that the blink rate represents a trade-off between gaining visual information through vigilance and the physiological benefits of blinking. We note that these results are only correlational, and we suggest that a link between vigilance and blinking could be demonstrated with suitable experimental manipulation of perceived risk (e.g. [8,39-41]).

We argued earlier that observed behaviours that are typically recorded as vigilance (such as holding a head upright or active scanning - see $[42,43]$ for discussion) may not be conducted solely for vigilance. Similarly, blinking may not solely be a maintenance behaviour that is traded-off against being able to collect visual information. Blinking may include a social element, as rhesus macaques Macaca mulatta are able entrain their blink rate in response to social cues, coordinating their blinking with partners that they were interacting with [44]. Our results suggest that the proportion of scanning time that an individual red deer

202 allocates to vigilance is related to the size of its immediate group, but we should

203 acknowledge that scanning behaviour may be influenced by social behaviour as well as 
204 vigilance. Earlier studies showed that young red deer were less vigilant than older ones

$205[32,33]$, and suggest this may be because the younger individuals are unlikely to be able to

206 outrun a predator if one appears. If the young in our study are following this behavioural

207 pattern, this also suggests that blinking may not be completely correlated with vigilance

208 level, as the younger deer had low blink rates when compared to mature adults. This may

209 reflect some form of social signalling between adults, although we note that we did not see a

210 difference between males and females (which contradicts previous results showing sex-

211 determined patterns of vigilance, where only females altered behaviour proportionally to

212 group size [32,33]). Other aspects of social behaviour may also be important for determining

213 blink rates, such as position within the group (echoing the vigilance changes seen in [33]).

214 Theory predicts that individuals on the outside of the group should be more vigilant than

215 those in the middle, and anecdotal evidence from olive baboons suggests that peripheral

216 individuals may blink less [31]. It may be possible to test many of the standard predictions

217 connecting vigilance and group size (e.g. [13-16]) using blink rate as a proxy for vigilance.

219 The blink rate may also be influenced by factors other than the size of the group, including

220 rainfall and wind (which have been shown to influence blink rate in captive grackles

221 Quiscalus mexicanus $[45,46])$. Similarly, the behaviour that an individual conducts

222 simultaneously to the blink may be important. Experiments in humans suggest a mechanism

223 controlling the timing of blinks, which occurs to minimise the chance of missing crucial

224 information [20,47-50], with evidence of similar behaviours in rhesus macaques Macaca

225 mulatta [44]. Peafowl Pavo cristatus also time their blinks to coincide with gaze shifts [51]

226 while grackles blink less during flight behaviours [52] and chickens blink more when feeding

227 when compared to scanning [30], all minimising the time where visual information cannot be

228 collected from the environment. Therefore, if an individual is moving, the timing and

229 frequency of its blinks may reflect this movement, and it may therefore be sensible to assay

230 blinking in response to group size in resting deer groups, which would not be undergoing

231 head and body movements that could confound the measure of blinking that is recorded.

232 Similarly, animals in different attentional states may change their frequency of blinking, such

233 as during sleep in herring gulls Larus argentatus [53].

235 Our results suggest that the measurement of blinking presents a simple and non-invasive

236 technique for observing attention that can be conducted remotely. Although we conducted

237 our sampling in the field, this could be done using video footage. Being able to analyse video

238 footage means that information about blink duration can also be collected, and previous

239 studies have demonstrated that this additional metric can also vary between individuals and

240 species [22,23,30,31,45,46], and may increase in relation to group size [30]. Given that 
bioRxiv preprint doi: https://doi.org/10.1101/2020.08.11.245837; this version posted January 30, 2021. The copyright holder for this preprint (which was not certified by peer review) is the author/funder, who has granted bioRxiv a license to display the preprint in perpetuity. It is made available under aCC-BY 4.0 International license.

241 blinking has been shown to decrease under stressful conditions [28,29], this simple

242 technique could help us to understand the welfare requirements of managed animals that

243 normally live in social groups.

244 


\section{ACKNOWLEDGMENTS}

ZWR and JHR conducted this work as their final year BSc projects. We thank the the School

of Biological Sciences teaching lab team for help with equipment, and two anonymous reviewers whose comments greatly improved the manuscript,

\section{FUNDING}

253

SAR was supported a Natural Environment Research Council (UK) standard grant

255

(NE/P012639/1).

256

257

258

\section{REFERENCES}

1. Krause J, Ruxton GD. 2002 Living in groups. Oxford: Oxford University Press.

2. Ward A, Webster M. 2016 Sociality: the behaviour of group-living animals. Switzerland: Springer.

3. Bednekoff PA, Lima SL. 1998 Re-examining safety in numbers: interactions between risk dilution and collective detection depend upon predator targeting behaviour. Proc $R$ Soc B 265, 2021-2026. (doi:10.1098/rspb.1998.0535)

4. Hamilton WD. 1971 Geometry for the selfish herd. J Theor Bio/ 31, 295-311. (doi:10.1016/0022-5193(71)90189-5)

5. Pulliam HR. 1973 On the advantages of flocking. J Theor Bio/ 38, 419-422. (doi:10.1016/0022-5193(73)90184-7)

6. Lima SL. 1990 The influence of models on the interpretation of vigilance. In Interpretation and explanation in the study of animal behavior, volume 2: explanation, evolution and adaptation (eds M Bekoff, D Jamieson), pp. 246-267. Boulder: Westview Press.

7. McNamara JM, Houston Al. 1992 Evolutionarily stable levels of vigilance as a function of group size. Anim Behav 43, 641-658.

8. Mónus F, Barta Z. 2016 Is foraging time limited during winter? - a feeding experiment with tree sparrows under different predation risks. Ethology 122, 20-29. (doi:10.1111/eth.12439)

9. Carere C, Montanino S, Moreschini F, Zoratto F, Chiarotti F, Santucci D, Alleva E. 2009 Aerial flocking patterns for wintering starlings, Sturnus vulgaris, under different predation risk. Animal Behaviour 77, 101-107. (doi:10.1016/j.anbehav.2008.08.034) 
282

283

284

285

286

287

288

289

290

291

292

293

294

295

296

297

298

299

300

301

302

303

304

305

306

307

308

309

310

311

312

313

314

315

316

317

318

319

320

10. Rands SA, Cowlishaw G, Pettifor RA, Rowcliffe JM, Johnstone RA. 2003 The spontaneous emergence of leaders and followers in a foraging pair. Nature 423, 432434. (doi:10.1038/nature01630)

11. Rands SA. 2011 Approximating optimal behavioural strategies down to rules-of-thumb: energy reserve changes in pairs of social foragers. PLoS One 6, e22104. (doi:10.1371/journal.pone.0022104)

12. Fernández-Juricic $E$, Smith R, Kacelnik A. 2005 Increasing the costs of conspecific scanning in socially foraging starlings affects vigilance and foraging behaviour. Anim. Behav. 69, 73-81. (doi:10.1016/j.anbehav.2004.01.019)

13. Beauchamp G. 2019 On how risk and group size interact to influence vigilance. Biol Rev 94, 1918-1934. (doi:10.1111/brv.12540)

14. Beauchamp G. 2008 What is the magnitude of the group-size effect on vigilance? Behav Ecol 19, 1361-1368. (doi:10.1093/beheco/arn096)

15. Beauchamp G. 2003 Group-size effects on vigilance: a search for mechanisms. Behav Process 63, 111-121.

16. Elgar MA. 1989 Predator vigilance and group size in mammals and birds: a critical review of the empirical evidence. Biol Rev 64, 13-33. (doi:10.1111/j.1469185X.1989.tb00636.x)

17. Allan ATL, Hill RA. 2018 What have we been looking at? A call for consistency in studies of primate vigilance. Am J Phys Anthropol 165, 4-22. (doi:10.1002/ajpa.23381)

18. Treves A. 2000 Theory and method in studies of vigilance and aggregation. Anim Behav $60,711-722$.

19. Blount WP. 1927 Studies of the movements of the eyelids of animals: blinking. Q J Exp Physiol 18, 111-125. (doi:10.1113/expphysiol.1927.sp000426)

20. Ranti C, Jones W, Klin A, Shultz S. 2020 Blink rate patterns provide a reliable measure of individual engagement with scene content. Sci Rep 10, 8267. (doi:10.1038/s41598020-64999-x)

21. Stevens JR, Livermore A. 1978 Eye blinking and rapid eye movement: pulsed photic stimulation of the brain. Exp Neurology 60, 541-556. (doi:10.1016/0014-4886(78)900092)

22. Tada H, Omori Y, Hirokawa K, Ohira H, Tomonaga M. 2013 Eye-blink behaviors in 71 species of primates. PLoS One 8, e66018. (doi:10.1371/journal.pone.0066018)

23. Rands SA. 2021 Phylogenetically-controlled correlates of primate blinking behaviour. PeerJ in press.

24. Kirsten SJ, Kirsten EB. 1983 Spontaneous blink rates of birds. Condor 85, 92-93.

25. Volkmann FC, Riggs LA, Moore RK. 1980 Eyeblinks and visual suppression. Science 207, 900-902. (doi:10.1126/science.7355270)

26. Riggs LA, Volkmann FC, Moore RK. 1981 Suppression of the blackout due to blinks. Vision Res 21, 1075-1079. (doi:10.1016/0042-6989(81)90012-2) 
27. Bristow D, Haynes J-D, Sylvester R, Frith CD, Rees G. 2005 Blinking suppresses the neural response to unchanging retinal stimulation. Curr Biol 15, 1296-1300. (doi:10.1016/j.cub.2005.06.025)

28. Cross DJ, Marzluff JM, Palmquist I, Minoshima S, Shimizu T, Miyaoka R. 2013 Distinct neural circuits underlie assessment of a diversity of natural dangers by American crows. Proc $R$ Soc B 280, 20131046. (doi:10.1098/rspb.2013.1046)

29. Merkies K, Ready C, Farkas L, Hodder A. 2019 Eye blink rates and eyelid twitches as a non-invasive measure of stress in the domestic horse. Animals 9, 562. (doi:10.3390/ani9080562)

30. Beauchamp G. 2017 Half-blind to the risk of predation. Front Ecol Evol 5, 131. (doi:10.3389/fevo.2017.00131)

31. Matsumoto-Oda A, Okamoto K, Takahashi K, Ohira H. 2018 Group size effects on interblink interval as an indicator of antipredator vigilance in wild baboons. Sci Rep 8, 10062. (doi:10.1038/s41598-018-28174-7)

32. Childress MJ, Lung MA. 2003 Predation risk, gender and the group size effect: does elk vigilance depend upon the behaviour of conspecifics? Anim Behav 66, 389-398. (doi:10.1006/anbe.2003.2217)

33. Lung MA, Childress MJ. 2007 The influence of conspecifics and predation risk on the vigilance of elk (Cervus elaphus) in Yellowstone National Park. Behav Ecol 18, 12-20. (doi:10.1093/beheco/arl066)

34. Clutton-Brock TH, Guinness FE, Albon SD. 1982 Red deer: behavior and ecology of two sexes. Chicago: Chicago University Press.

35. Rands SA, Muir H, Terry NL. 2014 Red deer synchronise their activity with close neighbours. PeerJ 2, e344. (doi:10.7717/peerj.344)

36. Mitchell B, Staines BW, Welch D. 1977 Ecology of red deer: a research review relevant to their management in Scotland. Cambridge: Institute of Terrestrial Ecology.

37. Rands SA. 2015 Nearest-neighbour clusters as a novel technique for assessing group associations. Royal Society Open Science 2, 140232. (doi:10.1098/rsos.140232)

38. R Development Core Team. 2019 R: a language and environment for statistical computing. Vienna: R Foundation for Statistical Computing.

39. Fey K, Banks PB, Ylönen H, Korpimäki E. 2010 Behavioural responses of voles to simulated risk of predation by a native and an alien mustelid: an odour manipulation experiment. Wildl Res 37, 273-282. (doi:10.1071/WR08031)

40. Abbey-Lee RN, Mathot KJ, Dingemanse NJ. 2016 Behavioral and morphological responses to perceived predation risk: a field experiment in passerines. Behav Ecol 27, 857-864. (doi:10.1093/beheco/arv228)

41. Rands SA, Cuthill IC. 2001 Separating the effects of predation risk and interrupted foraging upon mass changes in the blue tit Parus caeruleus. Proceedings of the Royal Society B 268, 1783-1790. (doi:10.1098/rspb.2001.1653) 
360
42. Mónus F. 2018 Competing activities as measures of fear and vigilance. Anim Sentience 2. (doi:10.51291/2377-7478.1312)

43. Beauchamp G. 2015 Animal vigilance: monitoring predators and competitors. London: Academic Press. See https://doi.org/10.1016/C2014-0-01423-2.

44. Ballesta S, Mosher CP, Szep J, Fischl KD, Gothard KM. 2016 Social determinants of eyeblinks in adult male macaques. Sci Rep 6, 38686. (doi:10.1038/srep38686)

45. Yorzinski JL, Argubright S. 2019 Wind increases blinking behavior in great-tailed grackles (Quiscalus mexicanus). Front Ecol Evol 7, 330. (doi:10.3389/fevo.2019.00330)

46. Yorzinski JL. 2020 Blinking behavior in great-tailed grackles (Quiscalus mexicanus) increases during simulated rainfall. Ethology 126, 519-527. (doi:10.1111/eth.13003)

47. Nakano T, Yamamoto Y, Kitajo K, Takahashi T, Kitazawa S. 2009 Synchronization of spontaneous eyeblinks while viewing video stories. Proc $R$ Soc B 276, 3635-3644. (doi:10.1098/rspb.2009.0828)

48. Shin YS, Chang W, Park J, Im C-H, Lee SI, Kim IY, Jang DP. 2015 Correlation between inter-blink interval and episodic encoding during movie watching. PLoS One 10, e0141242. (doi:10.1371/journal.pone.0141242)

49. Wiseman RJ, Nakano T. 2016 Blink and you'll miss it: the role of blinking in the perception of magic tricks. PeerJ 4, e1873. (doi:10.7717/peerj.1873)

50. Maffei A, Angrilli A. 2019 Spontaneous blink rate as an index of attention and emotion during film clips viewing. Physiol Behav 204, 256-263.

(doi:10.1016/j.physbeh.2019.02.037)

51. Yorzinski JL. 2016 Eye blinking in an avian species is associated with gaze shifts. Sci Rep 6, 32471. (doi:10.1038/srep32471)

52. Yorzinski JL. 2020 A songbird inhibits blinking behaviour in flight. Biol Lett 16, 20200786. (doi:10.1098/rsbl.2020.0786)

53. Amlaner CJ, McFarland DJ. 1981 Sleep in the herring gull (Larus argentatus). Anim Behav 29, 551-556. (doi:10.1016/S0003-3472(81)80118-2) 